\title{
Avoiding the diagnostic pitfalls of a pancreatic cystic lesion
}

\author{
RM Lukusa, ${ }^{1,2}$ (D) M Govender, ${ }^{1,2}$ (i) DL Clarke ${ }^{1,2}$ (D) \\ ${ }^{1}$ Department of Surgery, Greys Hospital, South Africa \\ ${ }^{2}$ Department of Surgery, Nelson R Mandela School of Medicine, University of KwaZulu-Natal, South Africa
}

Corresponding author, email: morgie@vodamail.co.za

\section{Summary}

Cystic pancreatic lesions present a diagnostic challenge. Accurate characterisation of an isolated cystic pancreatic lesion is therefore vital as this dictates the management strategy which may vary from radical resection to simple observation. This report describes how a detailed preoperative assessment led to the correct diagnosis of an isolated pancreatic lesion and appropriate safe and curative surgical management.

\section{Case presentation}

A 17-year-old female patient presented with a three month history of upper abdominal pain and progressive distention. She had no alarm symptoms, no comorbidities or previous surgical history and was of sober habits. She hailed from a rural community on the outskirts of Pietermaritzburg, not specifically a sheep-farming district but with sheep and cattle commonly encountered in the neighbourhood. On clinical examination, she was apyrexial, not jaundiced, not tachycardic and normotensive. Abdominal examination revealed an obvious large upper abdominal mass, extending from the epigastrium to just below the umbilicus. It was relatively firm, minimally tender and had a smooth regular surface.

Abdominal ultrasound showed a well-defined thick-walled septated cystic mass in the upper abdomen. It measured $20 \mathrm{x}$ $13 \mathrm{~cm}$ and was suspected to be arising from the left lobe of the liver. CT abdomen revealed a large intra-abdominal cyst with features of intracystic floating detached membranes (water-lily sign), originating from the tail of pancreas, suggestive of pancreatic hydatid cyst. There were no cysts in the liver or elsewhere in the abdomen (Figure 1). Hydatid serology was positive with a titre of 1:64. The rest of her blood results were normal including amoebic gel diffusion test, liver function tests, amylase, alpha feto-protein and CA 19-9.

The cyst's site and size were deemed inappropriate for radiological intervention. The patient was advised on the need for surgery. She was treated with the appropriate antihelminth, Albendazole $400 \mathrm{mg}$ twice daily for four weeks and then taken for laparotomy. Intraoperatively, a large thinwalled cyst in the lesser sac adherent to the posterior wall of stomach was identified. The cystic mass compressed the transverse colon but did not arise from it. There were no other lesions in the abdomen. After isolating the cyst with swabs soaked in hypertonic saline to minimise the risk of seeding, the cyst was aspirated and dissected down to the pancreas. It was densely adherent to the tail of the pancreas and the spleen. A distal pancreatectomy and splenectomy was performed to remove the cyst and its germinal layers in toto (Figure 2). A drain was left to the pancreatic bed.

The patient had an uneventful postoperative recovery. The drain was removed on day 4 post surgery with no evidence of a pancreatic fistula and the patient was discharged the following day. Histology confirmed a cystic mass measuring $21 \times 13 \times 2 \mathrm{~cm}$ with attached spleen and pancreas. Microscopy showed a cyst wall with pale laminations and intense PAS positivity. Within the peripancreatic tissue, there was a granulomatous response. The spleen was normal. The findings were in keeping with a pancreatic hydatid cyst.

The patient received pneumococcal vaccine 2 weeks postoperatively. Albendazole was continued for a further two months. She remained well on review at one, two and 6 months respectively. Repeat ultrasound at 3 months revealed no recurrence or cysts at other sites.

\section{Discussion}

Hydatid disease of the pancreas is very rare with a reported incidence between 0.1 and $2 \% .^{1-6}$ Although dogs are the definitive host and sheep and goats the intermediate hosts, humans can be accidentally infected by consuming contaminated offal. Dissemination is believed to be haematogenous or via the biliary system into the pancreatic duct through the lymphatics of the intestinal mucosa. ${ }^{3,4}$ The pancreatic head is the most frequent location of the cyst, followed by the tail. ${ }^{3-6}$ Chinya et al. and Sorogy et al. report a higher involvement of the body of pancreas rather than the tail. 7,8

The clinical presentation depends on cyst location and size, rather than the pathology. Cysts in the head of pancreas can present with obstructive jaundice. A cyst in body and tail of pancreas is usually asymptomatic but may present as an abdominal mass or cause symptoms due to compression. These patients often remain asymptomatic until the cyst becomes very large, as was the case with our patient. Patients may also present with complications such 


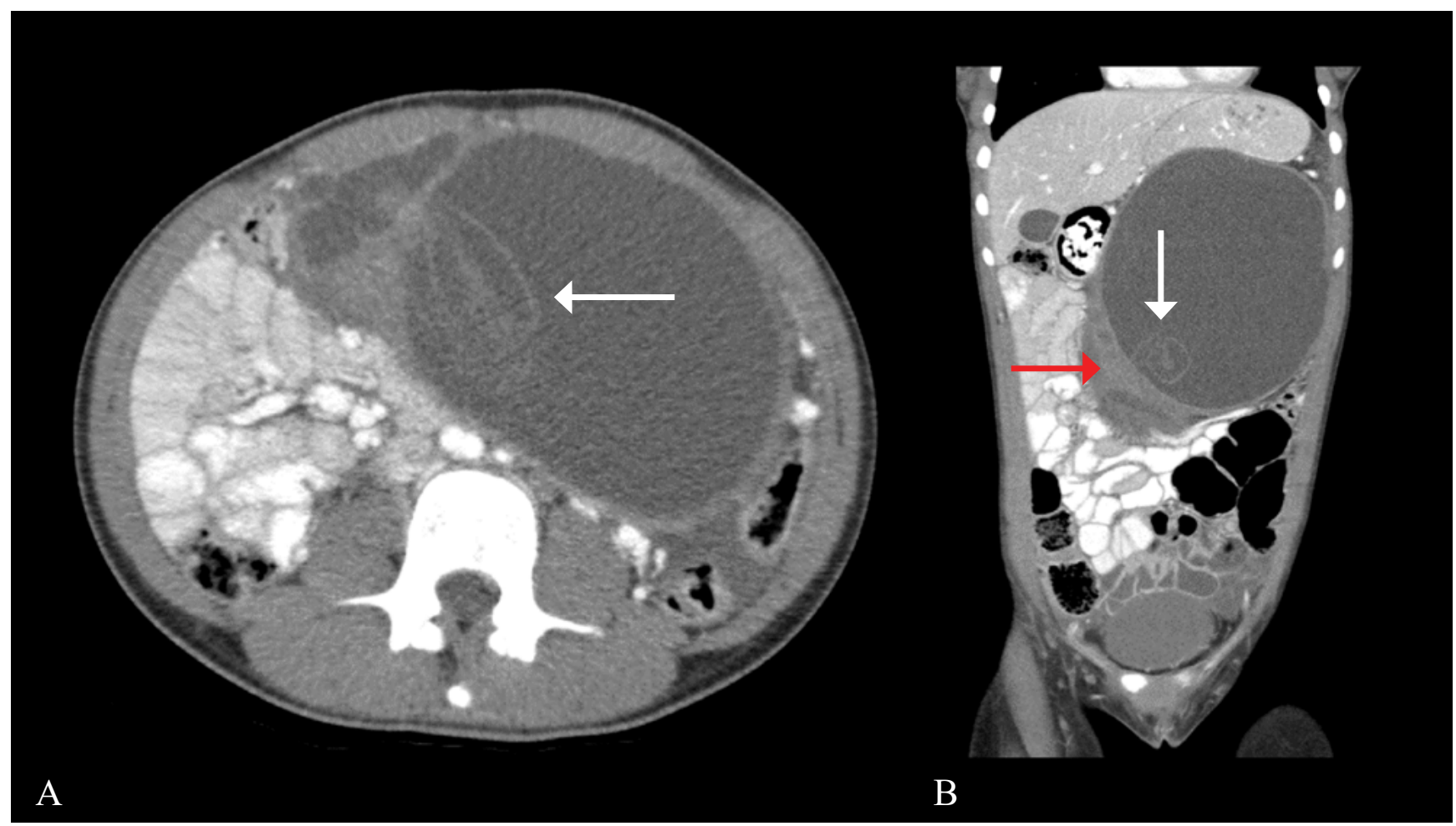

Figure 1A: Axial CT image demonstrating a well-defined cystic mass with intra-cystic floating membrane (water-lily sign) consistent with hydatid disease

Figure 1B: Coronal view demonstrating lesion arising from pancreas (red arrow) and water-lily sign (white arrow). The smaller cystic lesion noted in the right upper quadrant is the gallbladder

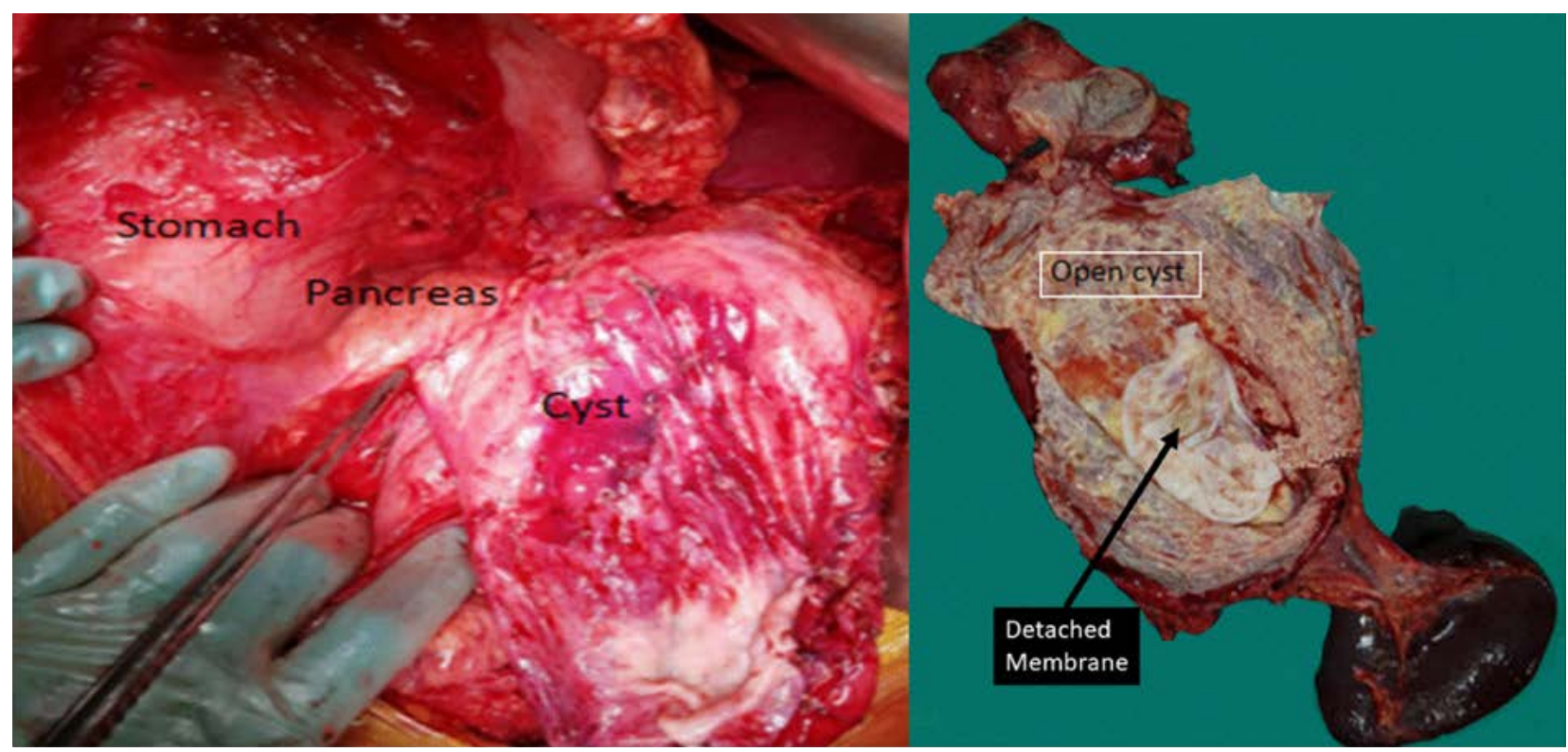

Figure 2A: Intraoperative image showing the dissected lesser sac following cyst aspiration and dissection to its base on the pancreas

Figure 2B: Resected specimen with cyst opened up showing detached membranes. Tail of pancreas and spleen can be seen on either side of the dissected cyst

as cholangitis, pancreatitis, splenic vein thrombosis and left sided portal hypertension. Epigastric pain appears to be the most common symptom. ${ }^{3,4,9}$

Cystic lesions of the pancreas have a wide differential. These include unilocular and multilocular cysts, with or without septation, debris or a solid component such as a pancreatic pseudocyst, mucinous cystadenoma (MCN), lymphoepithelial cyst, serous cystadenoma, solid pseudopapillary epithelial neoplasm, solid neoplasms with cystic degeneration and parasitic cysts.
This wide range of differentials can be narrowed down with careful history-taking, review of imaging characteristics and relevant serology. This patient from a rural community presented with a large macro-cystic lesion. The differentials could therefore be whittled down to pseudocyst, mucinous cystadenoma and hydatid disease. There was no preceding history of trauma or pancreatitis so pseudocyst was thought less likely. Given the young age of the patient and the lack of calcification or solid components within the cystic lesion, MCN was also considered less likely. Hydatid disease was 
therefore our most likely differential in this patient, and paired with the positive serology and characteristic features on imaging, a definitive diagnosis was reached.

Ultrasound can show the characteristic features of hydatid cyst namely hydatid sand, floating membranes (water-lily sign), daughter cysts and calcification of the cyst wall. However, ultrasound, though pathognomic in liver hydatids, is somewhat limited by the retroperitoneal position of the pancreas. ${ }^{3,4,9} \mathrm{CT}$ scan will show detailed cyst characteristics: cyst location, size, presence of floating membranes and/or daughter cysts and relation to pancreato-biliary system. ${ }^{3,4}$ MRI and MRCP are particularly important to delineate the relationship between the cyst and pancreatic and bile ducts, especially when the cyst involves the head of pancreas and/ or causes compression of the ducts. ${ }^{3,4}$ An MRI/ MRCP is of less significance in lesions of the distal pancreas. Enzymelinked immunosorbent assay for echinococcal antigens is positive in more than $85 \%$ of cases. ${ }^{4,7}$

Although surgery is the mainstay of treatment, certain precautions and caveats must be borne in mind. Albendazole (10 mg/Kg/day 12-hourly) should be given before surgery to reduce the risks of anaphylaxis and postoperative recurrence secondary to intraoperative spillage of cyst content, and it should continue postoperatively., ${ }^{3,4,9}$ The duration of this prophylactic antihelminthic treatment is variable in the literature.

Surgical options depend on the location of the cyst as well as whether there is pancreatic duct disruption. The recommended surgical options involve deroofing if no main pancreatic duct communication is demonstrated, or resection if there is a communication. As we were unable to separate the cyst from the tail of the pancreas in our patient and had not excluded a pancreatic duct fistula with MRI preoperatively, the decision was made to perform a distal pancreatectomy.

If dealing with a large cyst by deroofing, aspiration of the cyst should be performed followed by injection of a scolicidal agent which is then aspirated to dryness prior to cyst deroofing. We had aspirated the cyst to dryness to facilitate handling, but did not inject scolicidal agent as we were planning a resective and not a deroofing procedure. We had instead taken the precaution of isolating the aspirated cyst with swabs soaked in hypertonic saline.

\section{Conclusion}

Cystic lesions of the pancreas present a diagnostic challenge. Accurate diagnosis facilitates the selection of the appropriate management strategy. Primary hydatid cyst of the pancreas is a rare differential diagnosis. Its diagnosis requires the combination of a thorough history, clinical findings, hydatid serology and characteristic imaging. A resective surgical approach in conjunction with the appropriate anti-helminthic treatment was the correct approach for this giant cyst in the tail of the pancreas.

\section{Conflict of interest}

The authors declare no conflict of interest.

\section{Funding source}

No funding was required.

\section{Ethical approval}

The author/s declare that this submission is in accordance with the principles laid down by the Responsible Research Publication Position Statements as developed at the $2^{\text {nd }}$ World Conference on Research Integrity in Singapore, 2010 .

\section{ORCID}

RM Lukusa (D) https://orcid.org/0000-0002-7188-163X M Govender (ID https://orcid.org/0000-0002-4066-0156 DL Clarke (i) https://orcid.org/0000-0002-6954-153X

\section{REFERENCES}

1. Elaffand A, Vijay A, Mohamed S, et al. Echinococcal cyst of the pancreas with cystopancreatic duct fistula successfully treated by partial cystectomy and cystogastrostomy. J Surg Tech Case Rep. 2015;7(2):48-50.

2. Hiremath B, Subramaniam N, Boggavarapu M. Primary pancreatic hydatid cyst: an unexpected differential diagnosis. BMJ Case Rep. 2015;2015:bcr2015211377.

3. Akbulut S, Yavuz R, Sogutcu N, et al. Hydatid cyst of the pancreas: Report of an undiagnosed case of pancreatic hydatid cyst and brief literature review. World J Gastrointest Surg. 2014;6(10):190-200.

4. Ahmed Z, Chhabra S, Massey A, et al. Primary hydatid cyst of pancreas: case report and review of literature. Int J Surg Case Rep. 2016;27:74-7.

5. Kisaoglu A, Ozogul B, Atamanalp SS, Pirimoglu B, Aydinli B, Korkut E. Incidental isolated pancreatic hydatid cyst. Turkiye Parazitol Derg. 2015;39(1):75-7.

6. Yarlagadda $\mathrm{P}$, Yenigalla BM, Penmethsa U, Myneni RB. Primary pancreatic echinococcosis. Trop parasitol. 2013;3(2):151-4.

7. Chinya A, Khanolkar A, Kumar J, Sinha SK. Isolated hydatid cyst of the pancreas masquerading as pancreatic pseudocyst. BMJ Case Rep. 2015;2015:bcr2015211307.

8. Sorogy ME, El-Hemaly M, Aboelenen A. Pancreatic body hydatid cyst: a case report. Int J Surg Case Rep. 2015;6c:6870 .

9. Dziri C, Dougaz W, Bouasker I. Surgery of the pancreatic cystic echinococcosis: systematic review. Transl Gastroenterol Hepatol. 2017;2:105. 